\title{
Near unity Raman $\beta$-factor of surface enhanced Raman scattering in a waveguide
}

Ming Fu

Imperial College London

Mónica Mota

Imperial College London

Xiaofei Xiao

Imperial College London

Andrea Jacassi

Imperial College London

Yi Li

Southern University of Science and Technology

Ahad Riaz

Imperial College London

Stefan Maier

Imperial College

Rupert Oulton ( $\nabla$ r.oulton@imperial.ac.uk)

Imperial College London https://orcid.org/0000-0002-5070-3623

\section{Letter}

Keywords:

Posted Date: January 7th, 2022

DOI: https://doi.org/10.21203/rs.3.rs-1199041/v1

License: (c) (i) This work is licensed under a Creative Commons Attribution 4.0 International License. Read Full License

Version of Record: A version of this preprint was published at Nature Nanotechnology on October 27th, 2022. See the published version at https://doi.org/10.1038/s41565-022-01232-y. 


\title{
Near unity Raman $\beta$-factor of surface enhanced Raman scattering in a waveguide
}

\author{
Ming Fu ${ }^{1}$, Mónica P. dS. P. Mota ${ }^{1}$, Xiaofei Xiao ${ }^{1}$, Andrea Jacassi $^{1}, \mathrm{Yi} \mathrm{Li}^{1,2}$, Ahad Riaz $^{1}$, \\ Stefan A. Maier ${ }^{1,3}$, Rupert F. Oulton ${ }^{1, *}$

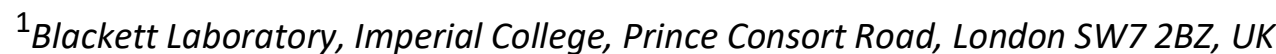 \\ ${ }^{2}$ School of Microelectronics, MOE Engineering Research Center of Integrated Circuits for Next Generation \\ Communications, Southern University of Science and Technology, Shenzhen 518055, China \\ ${ }^{3}$ Chair in Hybrid Nanosystems, Nanoinstitute Munich, Faculty of Physics, Ludwig-Maximilians-Universität \\ München, 80539 Munich, Germany \\ * Corresponding author: r.oulton@imperial.ac.uk
}

The Raman scattering of light by molecular vibrations offers a powerful technique to 'fingerprint' molecules via their internal bonds and symmetries. Since Raman scattering is weak ${ }^{1}$, methods to enhance, direct and harness it are highly desirable, e.g. through the use of optical cavities ${ }^{2}$, waveguides ${ }^{3-6}$, and surface enhanced Raman scattering (SERS) ${ }^{7-9}$. While SERS offers dramatic enhancements ${ }^{6,15,22,2}$ by localizing light within vanishingly small 'hot-spots' in metallic nanostructures, these tiny interaction volumes are only sensitive to few molecules, yielding weak signals that are difficult to detect ${ }^{10}$. Here, we show that SERS from 4Aminothiophenol (4-ATP) molecules bonded to a plasmonic gap waveguide is directed into a single mode with $>99 \%$ efficiency. Although sacrificing a confinement dimension, we find $>10^{4}$ times SERS enhancement across a broad spectral range enabled by the waveguide's larger sensing volume and nonresonant mode. Remarkably, the waveguide-SERS (W-SERS) is bright enough to image Raman transport across the waveguides exposing the roles of nanofocusing ${ }^{11-13}$ and the Purcell effect ${ }^{14}$. Emulating the $\beta$ factor from laser physics ${ }^{15-17}$, the near unity Raman $\beta$-factor observed exposes the SERS technique in a new light and points to alternative routes to controlling Raman scattering. The ability of W-SERS to direct Raman scattering is relevant to Raman sensors based on integrated photonics ${ }^{7-9}$ with applications in gas and biosensing as well as healthcare.

Raman spectroscopy has emerged as a powerful technique as it leverages the maturity of laser and detector technologies at visible wavelengths despite its meager efficiency. Various enhancement techniques relying on either stimulated Raman scattering ${ }^{1}$ or surface enhanced Raman scattering (SERS) ${ }^{18-20}$ have been developed. The stimulated Raman process underpins a range of powerful methods but relies on high intensity and short pulsed optical excitation, which can often damage samples. Meanwhile SERS ${ }^{21}$ has become a large field of research exploring metallic nanostructures capable of enhancing Raman by many orders of magnitude, for instance, rough metallic surface ${ }^{22}$, nanoparticles ${ }^{10,23,24}$, nano-gaps ${ }^{25,26}$, waveguides ${ }^{9,27}$, and metallic tips ${ }^{18,28,29}$.

Despite sensitivity to individual molecules, SERS has several limitations. Firstly, the strongest SERS requires vanishingly small 'hot-spots' where the enhancement is active yet only a few molecules may experience it. Secondly, resonant enhancements restrict the Raman bandwidth. Finally, SERS emerging from localized fields diffraction, making efficient detection difficult ${ }^{10}$. In this letter, we explore waveguide enhanced Raman scattering ${ }^{3-6}$, combined with $\mathrm{SERS}^{7-9}$, using the plasmonic waveguide, shown in Fig. 1a. It consists of a plasmonic gap waveguide with optical antenna couplers placed at either end ${ }^{30-32}$ on a glass substrate. Raman scattering is enhanced in the gap region by two mechanisms: the increased local excitation intensity by the nanofocusing effect ${ }^{11-13}$ and the Purcell effect ${ }^{14}$ due to enhanced vacuum fluctuations. Finite difference time domain (FDTD) simulation of the waveguide mode in Fig. 1b, shows the optical confinement strength. While the waveguide provides non-resonant SERS over many octaves, the enhancement persists here over the efficiency bandwidth of the antenna-waveguide coupling. Although this approach sacrifices confinement along one direction, strong waveguide-SERS (W-SERS) enables the imaging of Raman transport across a nanostructure and the observation of nanofocusing and Purcell effects. We find that SERS into the gap mode dominates as it drives the Purcell effect. Therefore, we introduce the spontaneous Raman $\beta$-factor ${ }^{15-17}$, to quantify the proportion of SERS coupling to this single mode. We show that W-SERS produces near unity Raman $\beta$-factors with $>10^{4}$ times enhancement across a broad spectral range. 
In this paper, we explore the Raman scattering of light by 4-ATP molecules ${ }^{33}$, which form a self-assembled monolayer on gold-air interfaces, as illustrated in Fig. 1a. The optical antennas were designed to provide in and out coupling via a high numerical aperture $(N A=1.45)$ oil immersion microscope system (see Methods) over a broad range of wavelengths. FDTD calculations used in the design process are described in Figure S1. The microscope system uses a wide-field imaging arrangement, where the focal spot of a pump laser can be scanned across the nanostructure, while the entire field of view is imaged in either Rayleigh or Raman scattering mode. Rayleigh imaging mode evaluates coupling and optical transport of pump light $\left(\lambda_{\mathrm{p}}=790\right.$ $\mathrm{nm})$ across the nanostructure. Meanwhile, Raman imaging mode not only reports Raman spectra $\left(\lambda_{\mathrm{s}}=800-\right.$ $900 \mathrm{~nm}$ ), but also resolves Raman photon emission and transport across the nanostructure.
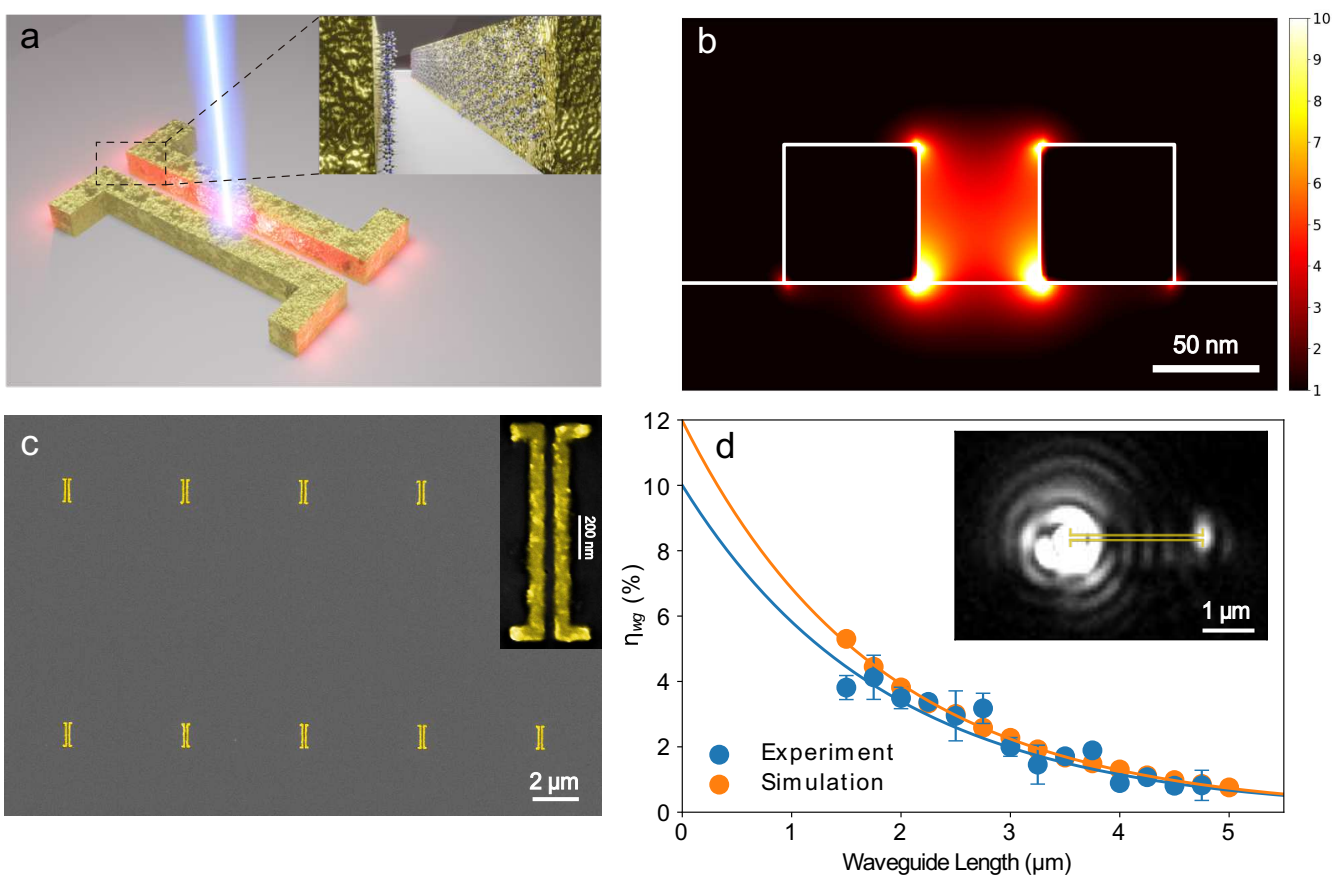

Fig 1. Illustration of plasmonic gap waveguides and their linear characterisation. a) Schematic of the plasmonic waveguide bonded with 4-Aminothiophenol (4-ATP) molecules. Under optical excitation of the plasmonic waveguide, most scattered Raman photons can be coupled into the waveguide as surface plasmon polariton (SPP) and coupled out through the antenna pairs at the end of waveguide. While only a few Raman photons can be coupled directly to the free space at the excitation position. b) Simulation of Electricity field intensity $|E|^{2}$ distribution at the cross section of waveguide. c) Scanning electron microscopic image of the waveguide. The inset panel shows one of the waveguide structures with higher resolution. d) The couple efficiency of waveguide $\eta_{w g}$, which is the ratio between coupled out laser intensity and incident laser intensity, as a function of the waveguide length. The blue dots and orange dots are experiment and simulation results respectively. The solid lines are the fitting results. The insert shows the focused laser excitation image of a waveguide with length of $2.5 \mu \mathrm{m}$. The excitation laser was tightly focused on the left side of the waveguide. The yellow line indicates the position of waveguide.

The plasmonic slot waveguides were constructed by two-step Electron Beam Lithography, as described in Methods. The high quality and reproducibility of the fabricated waveguides is evident from the Scanning Electron Microscope (SEM) image of an array of devices, shown in Figure 1c. From the inset of Fig. 1c, the waveguide structure is clearly shown: each plasmonic slot waveguide of length, $L$, and gap width, $G$, is decorated with dimer optical antennas, of length, $l$, on either end. To evaluate the coupling efficiency and propagation loss of these slot waveguides, we undertook a series of linear optical measurements on bare samples without the 4-ATP molecules. Light at a wavelength of $\lambda_{\mathrm{p}}=790 \mathrm{~nm}$ was focused to a diffraction 
limited spot with linear polarization parallel to the antenna to excite one of the antennas. Light coupled into the slot waveguide propagates to the other antenna, where it is imaged in Rayleigh scattering mode. Figure $1 \mathbf{d}$ shows the total coupling efficiency of waveguide, $\eta_{\mathrm{wg}}=\eta_{\text {antenna }} e^{-L / L_{\mathrm{sp}}}$, of light passing through the plasmonic waveguide for a range of different lengths, $L$, where $\eta_{\text {antenna }}=\eta_{\mathrm{in}} \eta_{\text {out }}$ is the net input and output coupling antenna efficiency, $L_{\mathrm{sp}}$ is the propagation length of the gap plasmon and the gap width, $G=60 \mathrm{~nm}$, and antenna length, $l=150 \mathrm{~nm}$ are fixed. The inset shows an image for $L=2.5 \mu \mathrm{m}$, where the larger spot is the reflection of the input beam at the input antenna, and the smaller spot is the scattering from the output antenna. Under the assumption that $\eta_{\text {in }}\left(\lambda_{\mathrm{p}}\right) \approx \eta_{\text {out }}\left(\lambda_{\mathrm{p}}\right)$ we determined both the antenna-waveguide coupling efficiency, $\eta_{\text {in }}\left(\lambda_{p}\right) \approx \sqrt{\eta_{\text {antenna }}}=30 \pm 2 \%$, and $L_{\text {sp }}\left(\lambda_{p}\right)=2.1 \pm 0.2 \mu m$. At Raman wavelengths, we find $\eta_{\text {out }}\left(\lambda_{\mathrm{s}}=860\right)=0.46 \pm 0.01$ (Figure S2) with sustained out-coupling efficiency achievable over the Raman emission band, as shown by simulations in Figure $\mathbf{S 1}$.

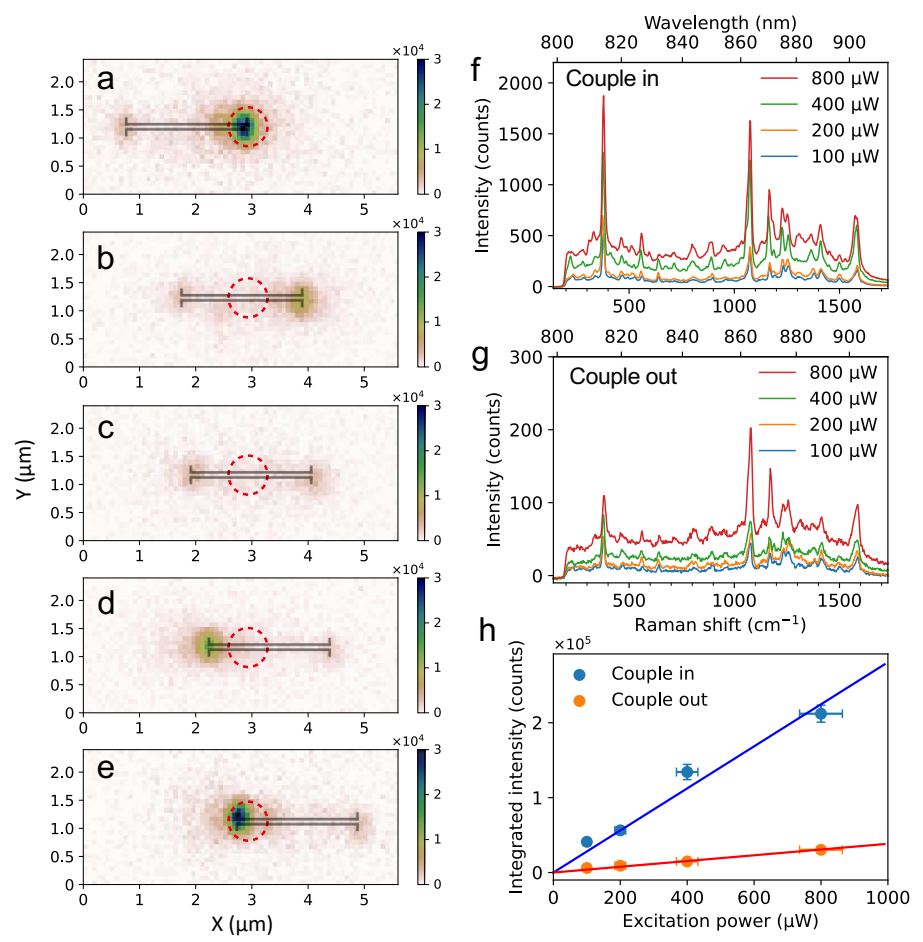

Fig 2. Imaging and spectroscopy of waveguide-based surface enhanced Raman scattering. a-e) Raman image of molecules bonded on the surface of plasmonic waveguide, by tightly focusing the laser on different position of the waveguide $(\mathrm{L}=2 \mu \mathrm{m})$. The dashed red circle indicates the position of the excitation laser spot. To guide the eye, the grey solid lines indicate the position of waveguide. f) and g) are the Raman spectra extracted from the left side (laser couple in) and right side (laser couple out) of waveguide with $\mathrm{L}=2 \mu \mathrm{m}$, with different excitation powers. h) Integrated Raman scattering intensity of laser couple in and couple out positions as a function of excitation power. The solid lines are the linear fitting results.

To exploit the enhancement and control of Raman scattering by the plasmonic waveguide, 4-ATP molecules were chemically bonded to the surface of the Au nanostructure. The details of the sample preparation are described in Methods. Figure 2 shows results of our experiment in Raman imaging mode for a pump wavelength of $\lambda=790 \mathrm{~nm}$. The series of images in Figs. 2a-e show the result of scanning the focal spot across the nanostructure from one antenna to the other. The brightest Raman emission occurs from an input antenna when the pump coincides with that antenna (Figs $\mathbf{2 a}$ and $\mathbf{2 e}$ ). The spectra of Raman scattering from both sides of the waveguide were measured by using a sensitive spectrometer, with spectra shown in Figs. $\mathbf{2 f}$ and $\mathbf{2 g}$. The broadband Raman scattering with featured Raman peaks of 4-ATP can be clearly observed from the spectra of both the input and output coupler positions. The integrated intensities of Raman scattering from both sides of the waveguide were found to be linearly dependent on the power of the excitation laser (Figure $\mathbf{2 h}$ ). In addition, we also measured 4-ATP molecules chemically bonded to a flat Au film, where a Raman spectrum 
was barely visible under the same experimental conditions. With a larger pump power and longer integration time, we were able to estimate that the plasmonic waveguide provided $10^{4}$ times more Raman scattering compared to the control sample (Figure S3).

The waveguide was then shifted from left to right, while the position of the focused laser beam was fixed, indicated by the dashed circle in the images of Figs. 2a-e. Raman scattering on the right-hand side gradually decreased when the waveguide was moved to the right, until eventually equal Raman emission was observed from both antennas when the laser beam was near the center of the waveguide (Figure 2c). With further displacement of the waveguide to the right, the reverse of the image in Figure $2 \mathbf{a}$ was observed, where the excitation beam is now on the left side of the waveguide (Figure 2e). The reversable position dependent Raman scattering shows the high precision of the home-built microscope and further confirms the high quality of the fabricated waveguides.

There are two key observations to draw from this experiment. Firstly, the Raman scattering is clearly emanating from regions not directly excited by the pump laser in all images in Figure 2. In Figs. 2a and 2e, transport is observed over a distance of $2 \mu \mathrm{m}$ from the diffraction limited excitation spot, which has $e^{-2}$ diameter of $0.82 \lambda_{\mathrm{p}} / N A \approx 447 \mathrm{~nm}$. Secondly, very little Raman scattering emanates from the position of the excitation beam in Figure $\mathbf{2 c}$ when the waveguide is centrally pumped, Raman scattering is only observed at the output couplers. This is evidence of the strengthened vacuum fluctuations of the slot waveguide, where Raman scattering into the waveguide is accelerated compared to Raman scattering into radiation modes of free space. This causes Raman scattering into the waveguide modes to dominate over that scattered as radiation leading to a high Raman $\beta$-factor. The broadband nature of this enhancement mechanism explains why no Raman, or indeed gold fluorescence, is observed at the position of the excitation beam.
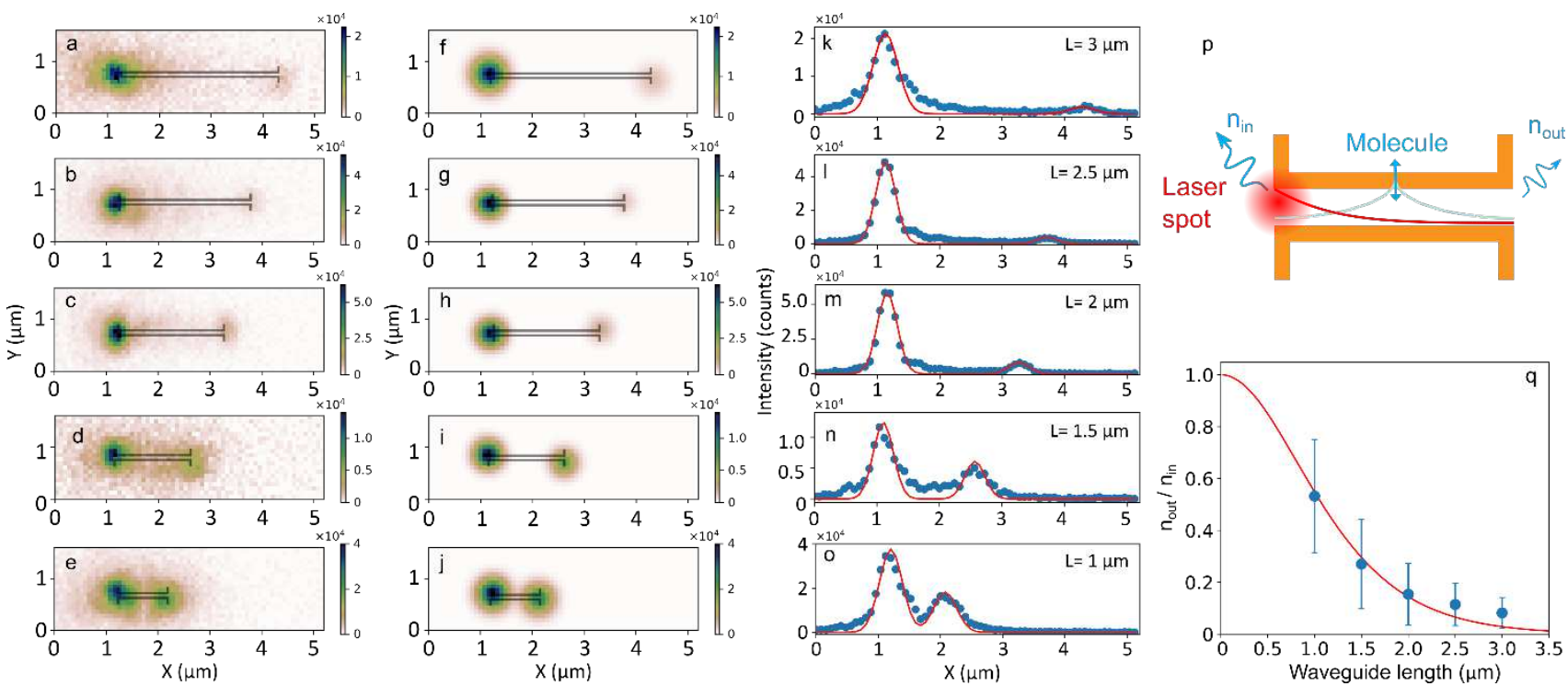

Fig 3. Imaging of Raman scattering transport in W-SERS. a-e) The Raman image of molecules bonded to waveguide with different lengths, with the excitation beam tightly focused on the left side of the waveguide. $\mathrm{f}$ j) The two-dimensional Gaussian fittings of the a-e). To guide the eye, the grey solid lines indicate the position of waveguide. $k-0$ ) the image profile of a-e) and $\mathrm{f}-\mathrm{j}$ ). p) The scheme of laser propagation along the waveguide and Raman scattering of molecules. $n_{\text {in }}$ and $n_{\text {out }}$ are the Raman photons collected from laser couple in and couple out positions. q) The ratio of Raman photons collected from laser couple in and couple out positions $n_{\text {out }} / n_{\text {in }}$, with laser illuminated at the antennas. The red solid line is the theoretical result.

To explore the mechanisms of Raman scattering in the plasmonic gap waveguides, the excitation laser was positioned on the left-hand antenna of waveguides of varying length, $L$, and Raman scattering images were collected. As observed in Figs $\mathbf{3}$ a-e, the Raman scattering is strongest at the position of the excitation laser, 
while the Raman scattering on the right-hand side decreases with increasing waveguide length. To quantitively determine the intensity of Raman scattering on both sides of the waveguide, the Raman images were fit to 2D Gaussian functions, as shown in Figs $\mathbf{3} \mathbf{f}-\mathbf{j}$, where the matching profiles of raw and fitted images confirm the quality of the fits (Figs $\mathbf{3} \mathbf{k}-\mathbf{o}$ ). We have developed a theoretical model that considers the bi-directional Raman scattering into the waveguide mode as shown in the scheme of Figure $3 p$ (see Methods). For excitation at the left-hand antenna at position $x=0$, for a waveguide of length $L$, the ratio of the Raman photon numbers from the input, $n_{\text {in }}$, and the output, $n_{\text {out }}$, antennas is,

$$
\frac{n_{\text {out }}}{n_{\text {in }}}=\bar{L}[\sinh \bar{L}]^{-1},
$$

where $\bar{L}=L / L_{\mathrm{sp}}$, is the ratio of waveguide and mode propagation lengths and is the only free parameter. The model matches well with the experimental data (Figure 3q and Figure S5) for a propagation length of $0.7 \pm$ $0.1 \mu \mathrm{m}$, which is about $2.6 \times$ smaller than the value obtained from Rayleigh imaging measurements, in Fig $\mathbf{1 d}$. To confirm this modified value of $L_{s p}$, extracted from the Raman model, we directly measured $L_{s p}$ again for the samples coated with 4-ATP using Rayleigh scattering imaging mode. We find that the 4-ATP introduces additional absorption, reducing propagation length, but not affecting the coupling efficiency (Figure S2). The role of molecular absorption on Raman scattering is clearly shown in this waveguide setting and indicates the length scales over which Raman scattering can be effectively collected. In this case, the total Raman signal is maximized for increasing slot waveguide length up to about $L=2 L_{s p} \approx 1.4 \mu \mathrm{m}$ (see Section S4 in Supplementary Information), which presents a substantial increase in interaction volume compared to other SERS approaches. For molecules that absorb less pump light, greater interaction lengths would be possible.

Alternatively, when the waveguide is illuminated centrally, we consider the total number of Raman photons collected from both antennas, $n^{(c)}$. When normalized against $n_{\text {in }}$, the number of photons emitted from the input antenna for the antenna-coupled case, we find a ratio

$$
\frac{n_{\mathrm{in}}}{n^{(c)}}=\left(\frac{A_{0}}{A_{\mathrm{wg}}\left(\lambda_{\mathrm{p}}\right)}\right) \frac{\eta_{\mathrm{in}}\left(\lambda_{\mathrm{p}}\right) n_{\mathrm{g}}\left(\lambda_{\mathrm{p}}\right) L_{\mathrm{sp}}}{4 \sqrt{2 \pi} \rho H} e^{\bar{L} / 2},
$$

where $\rho=0.41 \lambda_{\mathrm{p}} / N A$ is the radius of the Gaussian beam used to excite the waveguide, both centrally and at the antenna, $A_{0}=\pi \rho^{2}$ is the beam area, and $H$ is the local electric field enhancement of the Gaussian beam due to the waveguide's narrow gap. $H$ was evaluated numerically as described in Figure S4. The experimentally measured ratio is fit to the function, $n_{\mathrm{in}} / n^{(c)}=a e^{L / 2 L_{\mathrm{sp}}}$, for varying waveguide length, $L$, as shown in Figure S6. The value $a=0.97 \pm 0.01$ allows us to determine experimentally the plasmonic waveguide mode area, and the average Stoke's field propagation length, $L_{\mathrm{sp}}\left(\lambda_{\mathrm{s}}\right)=0.780 \pm 0.004 \mu \mathrm{m}$. The mode area reduction, $A_{0} / A_{\mathrm{wg}}\left(\lambda_{\mathrm{p}}\right)=14.94 \pm 0.96$, compares well with the simulated value of 14.68 (Table S1). Notably, the Raman signal from antenna excitation always dominates over central excitation.

We can use this estimate of the waveguide mode area to determine the average pump intensity enhancement, $I_{\mathrm{p} 0} / I_{\mathrm{f} 0}=84.45 \pm 0.87$, relative to the peak Gaussian beam intensity, $I_{\mathrm{f} 0}$, of the gold film control sample. When multiplied with a numerical estimate for the Raman Purcell enhancement factor of $F_{\mathrm{p}}=42.55$, we find the total Raman enhancement factor of 7,504 \pm 78 , which compares well to the observed enhancement relative to the gold film control sample of $13,032 \pm 227$ (Figure S3). 

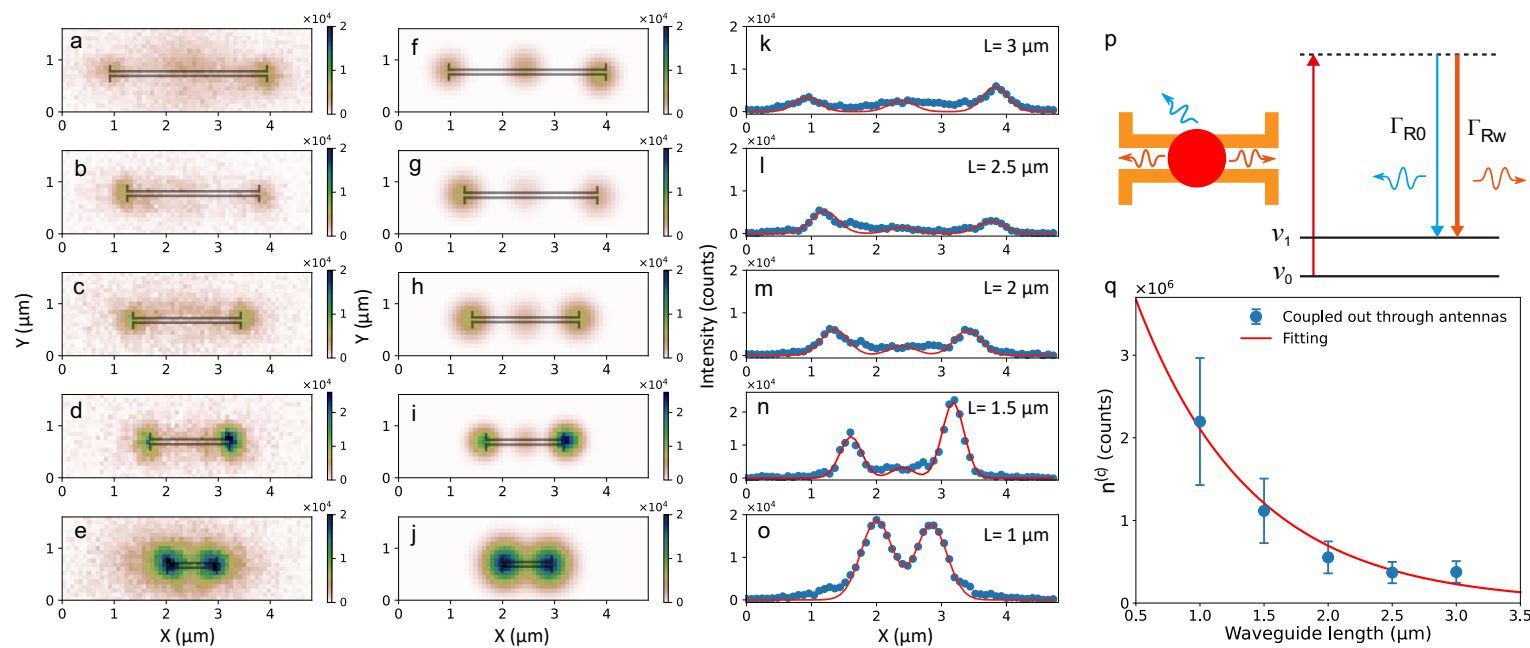

Fig 4. Experimental evaluation of the Raman $\boldsymbol{\beta}$ factor. a-e) The Raman image of molecules bonded to waveguide with different lengths, with the excitation beam tightly focused on the middle of the waveguide. $\mathrm{f}-\mathrm{j}$ ) The two-dimensional Gaussian fittings of the a-e). The grey solid lines indicate the position of waveguide. k-o) the image profile of a-e) and $f-j$ ). p) The scheme of Raman scattering coupled to free space and to the waveguide as surface plasmon polariton, with scattering rate of $\Gamma_{\mathrm{R} 0}$ and $\Gamma_{\mathrm{Rw}}$. q) The intensity of Raman scattering coupled out through two antenna pairs $n^{(c)}$ as a function the waveguide length. The red solid line the exponential fitting.

We now continue with the centrally illuminated waveguide case and consider the proportions of Raman photons emitted directly as radiation and emitted from the antennas via the waveguide mode. Let us assume that the 4-ATP molecules excited by the pump beam scatter Raman photons directly as radiation at a rate, $\Gamma_{\mathrm{R} 0}$, and into the waveguide mode with rate $\Gamma_{\mathrm{Rw}}$, as illustrated in the schematic of Figure $4 \mathrm{p}$. Using our Raman imaging approach, we can now estimate the Raman $\beta$-factor, where $\beta \approx \Gamma_{\mathrm{Rw}} /\left(\Gamma_{\mathrm{Rw}}+\Gamma_{\mathrm{R} 0}\right)$. To estimate to Raman $\beta$-factor, we measured the Raman photons directly coupled to free space at the excitation position, $n_{\mathrm{R} 0}^{(c)} \propto \Gamma_{\mathrm{R} 0}$, and compare this with the total Raman photons collected at the antennas, $n^{(c)}$. The number of Raman photons emitted directly into the waveguide mode at the excitation position, $n_{\mathrm{Rw}}^{(c)} \propto \Gamma_{\mathrm{Rw}}$, are related to $n^{(c)}$ through the propagation loss to the antennas and their out coupling efficiency, where $n^{(c)}=$ $\eta_{\text {out }}\left(\lambda_{\mathrm{s}}\right) n_{\mathrm{Rw}}^{(c)} e^{-\bar{L} / 2}$. We thus estimate the Raman $\beta$-factor using,

$$
\beta=\left[1+\frac{n_{\mathrm{R} 0}^{(c)}}{n_{\mathrm{RW}}^{(c)}}\right]^{-1}
$$

From the fitting, shown in Figure $\mathbf{4 q}$, we found $\beta=0.993 \pm 0.001$ so that with a high degree of certainty, less than $1 \%$ of the Raman emitted at the excitation spot radiate directly. We can check this value of $\beta$ against our calculated Purcell factor of $F_{p}=42.55$, with $\beta \approx\left(F_{p}-1\right) / F_{p}=0.98$ (see Methods). Raman scattering is therefore clearly bidirectional emission with near unity efficiency and couples to a single waveguide mode. We note that a single mode silica optical fiber would have a Raman $\beta \sim 10^{-3}$. As is seen in low threshold lasers, the Raman $\beta$ factor determines the strength of stimulated Raman scattering. We thus propose that the W-SERS approach reduces the threshold of stimulated Raman scattering by many orders of magnitude.

W-SERS enables strong Raman enhancement due to lateral confinement, with the added ability to efficiently collect the scattering in a waveguide with near unity Raman $\beta$-factor. Although we observed $>10^{4}$ times enhancement, the enhancement scales dramatically with reducing gap width so that $>10^{6}$ is possible for a gap width of $10 \mathrm{~nm}^{25}$ (Figure S7). Here we have seen Raman scattering from the relatively sparse distribution of 4-ATP molecules bound to the gold surface; however, the gap region presents a substantial sensing volume with the opportunity for stronger optical-molecule interactions. The W-SERS technique also provides new 
insight into the SERS mechanism and in particular the role of the Raman $\beta$-factor. We have proposed that this could reduce the threshold for stimulated SERS by many orders of magnitude, a route to improved Raman sensing. The ability for W-SERS to direct Raman scattering with a high Raman $\beta$-factor could be exploited for highly sensitive on-chip Raman sensor based on integrated photonics with applications in gas and bio-sensing as well as healthcare applications.

\section{Methods:}

\section{Sample preparation}

\section{Fabrication of Antenna/Waveguide}

The plasmonic waveguides were fabricated by electron beam lithography (EBL) on $175 \mu \mathrm{m}$ thick glass substrates. The substrate was submerged for 24 hours in a solution of DECON and water (1:100), and then dipped in acetone, sonicated, rinsed with IPA, dried with nitrogen and plasma ashed with oxygen for 5 min. A polymer, resist PMMA 475, was spin-coated on the glass substrate (3500 rpm, $1 \mathrm{~min}$ ) and baked at $180 \mathrm{C}$ for $5 \mathrm{~min}$. On top of the first polymer layer a second one of PMMA 950 is spin-coated and then baked with the same parameters of the first one. To guarantee a high conductivity of the sample, a layer of E-Spacer was spincoated as well (2000 rpm, $1 \mathrm{~min}$ ) and baked $\left(90^{\circ} \mathrm{C}, 1 \mathrm{~min}\right)$. A Raith Nanofabrication Electron Micoscope was used to perform the electron lithography. A lift-off process was performed to remove the polymer and the gold on top of it. The sample was sunk into acetone for 24 hours then rinsed with IPA and dried with nitrogen. To create the most reproducible gaps, a two-step method was employed for each half of the waveguide design using the same steps described above. For alignment of the two EBL exposures, markers were created during the first step and an automatic alignment procedure guaranteed excellent matching of the structures.

\section{Coating 4-ATP molecules on gold waveguides}

Before coating 4-ATP molecules on gold waveguides, the waveguides sample was rinsed with Acetone and IPA. Then the sample was dried out with $\mathrm{N}_{2}$ flow. 4-ATP solution was prepared by dispersing the 4-ATP molecules in ethanol. The waveguides sample was further cleaned in $\mathrm{O}_{2}$ plasma for more than 1 minute. Then the sample was immediately dropped into the prepared 4-ATP ethanol solution and kept in the solution overnight. Lastly, the sample was rinsed with ethanol before the measurements.

\section{Optical Characterization of Waveguides}

The individual waveguide structures with and without 4-ATP molecules were characterised by a home-made wide-field optical microscope system. The coherent light from a tuneable continuous-wave Ti-Sapphire laser system (Model 3900, Spectra-Physics) was tightly focused onto the sample with diffraction limited spot size by an oil objective lens (Plan Apo Lambda, $100 \times, N A=1.45$, Nikon). A half waveplate was used to adjust the beam's polarisation to be parallel to the antennas of the waveguides. A short-pass filter (FESH0800, Thorlabs) was used to clean the excitation laser frequency. For laser scattering measurement, a wedge beam splitter was used to reflect the laser beam to the objective lens whilst allowing the laser scattering signal from the sample transmits through it with high efficiency. For Raman image and spectra measurements, a long pass dichroic beam splitter (FF801-Di02-25×36, Semrock) was used to reflect the laser beam while the Raman scattering signal can transmit through efficiently. The sample was mounted on a 3-dimensional open loop piezo stage (3-Axis NanoMax, Thorlabs) with stepper motor (MAX383, Thorlabs). One to two drops of immersion oil with refractive index $n=1.45$ (Nikon type $N$ ) were placed in between the substrate and the objective lens to minimise the refractive index mismatch.

For navigation purposes, a white light source (QTH10, Thorlabs) illuminated the side of the waveguide structures, so that their images and the transmitted white light could be collected on the other side by a camara (webcam, Logitech). A long pass filter (BLP01-808R-25, Semrock) and a notch filter (NF785-33, Thorlabs) were placed to block the laser scattering for Raman image and spectra measurement. An electron multiplying 
charge coupled device (EMCCD) (Rolera EM-C ${ }^{2}$ ) was placed on the image plane to image the laser excitation image and Raman image. The Raman photons are sent to a spectrometer (Acton 300, Princeton Instrument) combined with a cooled CCD camera (PIXIS 100, Princeton Instrument) to measure the Raman spectra.

\section{Waveguide Enhanced Raman Theory}

We consider Raman scattering in a waveguide using a scalar 1D differential equation in the number of scattered Raman photons ${ }^{34}$, described in detail in the Supplementary Information. The generated Stoke's Raman photon numbers along the waveguide, $n_{\mathrm{f}}(z)$ and $n_{\mathrm{b}}(z)$, in the forwards and backwards directions are determined by the $1 \mathrm{D}$ differential equations,

$$
\begin{aligned}
& \frac{d n_{\mathrm{f}}(z)}{d z}=-\alpha_{\mathrm{s}} n_{\mathrm{f}}(z)+g_{\mathrm{Rw}} I_{\mathrm{p}}(z) \\
& \frac{d n_{\mathrm{b}}(z)}{d z}=\alpha_{\mathrm{s}} n_{\mathrm{b}}(z)-g_{\mathrm{Rw}} I_{\mathrm{p}}(z)
\end{aligned}
$$

where $g_{\mathrm{Rw}}$ is the forward and backward Raman scattering coefficient into the waveguide mode, and $\alpha_{\mathrm{s}}=L_{\mathrm{sp}}^{-1}$, is the absorption coefficient at the Stokes wavelength. Pump light is introduced either via waveguide coupling from the antenna, such that,

$$
I_{\mathrm{p}}(z)=I_{\mathrm{p} 0} e^{-\bar{L}}
$$

where $I_{\mathrm{p} 0}$ is the local peak pump intensity in the waveguide mode at the input coupler and $\bar{L}=\alpha_{\mathrm{s}} L=L / L_{\mathrm{sp}}$. In this case, the numbers of Raman photons emitted from each antenna coupler are,

$$
\begin{aligned}
& n_{\text {out }}=\eta_{\text {out }}\left(\lambda_{\mathrm{s}}\right) n_{\mathrm{f}}(L)=\eta_{\text {out }} \kappa L e^{-\bar{L}} \\
& n_{\text {in }}=\eta_{\text {out }}\left(\lambda_{\mathrm{s}}\right) n_{\mathrm{b}}(0)=\eta_{\text {out }}\left(\lambda_{\mathrm{s}}\right) \frac{\kappa}{2 \alpha_{\mathrm{s}}}\left(1-e^{-2 \bar{L}}\right)
\end{aligned}
$$

Where $\eta_{\text {out }}$ is the output coupling efficiency from the waveguide antennas at the Stoke's Raman wavelength and $\kappa=g_{\mathrm{Rw}} I_{\mathrm{p} 0}$. The ratio of Raman scattering from the two couplers is thus,

$$
\frac{n_{\text {out }}}{n_{\text {in }}}=\bar{L}[\sinh \bar{L}]^{-1}
$$

When the waveguide is illuminated centrally, we consider the following Gaussian intensity distribution,

$$
I_{\mathrm{p}}(z)=H I_{\mathrm{g} 0} e^{-2(z-L / 2)^{2} / \rho^{2}}
$$

where $\rho$ is the Gaussian beam radius and $I_{\mathrm{g} 0}$ is the peak intensity. The parameter, $H=4.29$, accounts for the local electric field enhancement of the Gaussian beam due to the waveguide's narrow gap and was evaluated numerically as described in the Supplementary Information. The total number of Raman photons collected from both antennas is,

$$
n^{(c)}=\eta_{\text {out }}\left(\lambda_{\mathrm{s}}\right)\left(n_{\mathrm{f}}^{(c)}(L)+n_{\mathrm{b}}^{(c)}(0)\right)=\eta_{\text {out }}\left(\lambda_{\mathrm{s}}\right) \sqrt{2 \pi} \rho \gamma H e^{\alpha_{\mathrm{s}}^{2} \rho^{2} / 8-\bar{L} / 2}
$$

where $\gamma=g_{\mathrm{Rw}} I_{\mathrm{g} 0}$. When comparing Raman scattered photons by the antenna and centre illumination methods, we account for the same incident power, $P_{0}$. For the waveguide mode, the coupling peak intensity to the waveguide is $I_{\mathrm{p} 0}=\eta_{\mathrm{in}}\left(\lambda_{p}\right) n_{\mathrm{g}}\left(\lambda_{\mathrm{p}}\right) P_{0} / A_{\mathrm{wg}}\left(\lambda_{\mathrm{p}}\right)$, where $n_{\mathrm{g}}\left(\lambda_{\mathrm{p}}\right)$ is the mode group index and $A_{\mathrm{wg}}\left(\lambda_{\mathrm{p}}\right)$ is the waveguide mode area at the pump wavelength. For the Gaussian beam intensity, $I_{\mathrm{g} 0}=2 P_{0} / A_{0}$, where 
$A_{0}=\pi \rho^{2}$. The ratio $n_{\mathrm{in}} / n^{(c)}$, which can be experimentally measured, allows us to determine the ratio of the Gaussian beam and waveguide mode areas:

$$
\frac{A_{0}}{A_{\mathrm{wg}}\left(\lambda_{\mathrm{p}}\right)}=\left(\frac{n_{\mathrm{in}}}{n^{(c)}}\right) 4 \sqrt{2 \pi} \alpha_{\mathrm{s}} \rho \frac{H}{\eta_{\mathrm{in}}\left(\lambda_{\mathrm{p}}\right) n_{\mathrm{g}}\left(\lambda_{\mathrm{p}}\right)} e^{-\bar{L} / 2}
$$

which agrees well with computed mode areas as shown in the Table S2. The Purcell factor at the Stoke's wavelength can be computed using the formula ${ }^{35-37}$,

$$
F_{\mathrm{pw}}=3 \frac{n_{\mathrm{g}}\left(\lambda_{s}\right)}{\pi} \frac{(\lambda / 2)^{2}}{A_{w g}\left(\lambda_{s}\right)}
$$

where $n_{\mathrm{g}}\left(\lambda_{\mathrm{s}}\right)$, the mode group index, and $A_{\mathrm{wg}}\left(\lambda_{\mathrm{s}}\right)$ must be computed numerically at the Stoke's wavelength. The total Purcell factor must also include the Raman scattering to radiation. Here we assume that Raman scattering to radiation on balance is not affected by the waveguide so that $F_{\mathrm{p}} \approx 1+F_{\mathrm{pw}}$. The estimation of the total Raman enhancement factor is discussed in the Supplementary Information. The fraction of Raman scattering directly into the waveguide mode (Raman $\beta$ factor), may be crudely approximated by,

$$
\beta \approx \frac{F_{\mathrm{pw}}}{1+F_{\mathrm{pw}}} \approx \frac{F_{p}-1}{F_{p}}
$$

which is often quoted in the literature in the case of spontaneous fluorescence ${ }^{15}$.

\section{Acknowledgements}

This work was supported by the EPSRC Reactive Plasmonics Programme (EP/M013812/1) and the Leverhulme Trust. This project has received funding from the European Union's Horizon 2020 research and innovation programme under the Marie Skłodowska-Curie grant agreement No 844591.

\section{Author Contributions}

M.F., M.P.dS.P.M. and R.F.O. developed the idea and designed the experiments. M.P.dS.P.M. and X.X. designed and simulated the waveguide structures and their mode properties. A.J. and Y.L. fabricated the waveguides and performed structural characterisation. M.F., M.P.dS.P.M. and Y.L. prepared for the sample with 4-ATP molecules. M.F. and M.P.dS.P.M. conducted the experiments and analysed the data. A.R. and R.F.O. developed the waveguide Raman model and analysed this in the context of the experimental data. M.F., M.P.dS.P.M., X.X., A.J., Y.L., A.R., S.A.M. and R.F.O. all contributed to the writing of the manuscript.

\section{References:}

1. Camp Jr, C. H. \& Cicerone, M. T. Chemically sensitive bioimaging with coherent Raman scattering. Nat.

Photonics 9, 295-305 (2015).

2. Hümmer, T. et al. Cavity-enhanced Raman microscopy of individual carbon nanotubes. Nat. Commun. 7, 12155 (2016).

3. Dhakal, A. et al. Nanophotonic Waveguide Enhanced Raman Spectroscopy of Biological Submonolayers.

ACS Photonics 3, 2141-2149 (2016). 
4. Holmstrom, S. A. et al. Trace gas Raman spectroscopy using functionalized waveguides. Optica 3, 891-896 (2016).

5. Eslami, S. \& Palomba, S. Integrated enhanced Raman scattering: a review. Nano Converg. 8, 41 (2021).

6. Ettabib, M. A. et al. Waveguide Enhanced Raman Spectroscopy for Biosensing: A Review. ACS Sens. 6, 2025-2045 (2021).

7. Wong, H. M. K., Dezfouli, M. K., Sun, L., Hughes, S. \& Helmy, A. S. Nanoscale plasmonic slot waveguides for enhanced Raman spectroscopy. Phys. Rev. B 98, 085124 (2018).

8. Turk, N. et al. Comparison of Free-Space and Waveguide-Based SERS Platforms. Nanomater. Basel Switz. 9, E1401 (2019).

9. Raza, A. et al. ALD assisted nanoplasmonic slot waveguide for on-chip enhanced Raman spectroscopy. APL Photonics 3, 116105 (2018).

10. Wang, D., Zhu, W., Best, M. D., Camden, J. P. \& Crozier, K. B. Directional Raman Scattering from Single Molecules in the Feed Gaps of Optical Antennas. Nano Lett. 13, 2194-2198 (2013).

11. Stockman, M. I. Nanofocusing of Optical Energy in Tapered Plasmonic Waveguides. Phys. Rev. Lett. 93, 137404 (2004).

12. Nielsen, M. P. et al. Adiabatic Nanofocusing in Hybrid Gap Plasmon Waveguides on the Silicon-onInsulator Platform. Nano Lett. 16, 1410-1414 (2016).

13. Nielsen, M. P., Shi, X., Dichtl, P., Maier, S. A. \& Oulton, R. F. Giant nonlinear response at a plasmonic nanofocus drives efficient four-wave mixing. Science 358, 1179-1181 (2017).

14. Purcell, E. M. Spontaneous Emission Probabilities at Radio Frequencies. in Confined Electrons and Photons: New Physics and Applications (eds. Burstein, E. \& Weisbuch, C.) 839-839 (Springer US, 1995). doi:10.1007/978-1-4615-1963-8_40.

15. van Exter, M. P., Nienhuis, G. \& Woerdman, J. P. Two simple expressions for the spontaneous emission factor $\beta$. Phys. Rev. A 54, 3553-3558 (1996).

16. Sorger, V. J. et al. Strongly Enhanced Molecular Fluorescence inside a Nanoscale Waveguide Gap. Nano Lett. 11, 4907-4911 (2011). 
17. Arcari, M. et al. Near-Unity Coupling Efficiency of a Quantum Emitter to a Photonic Crystal Waveguide. Phys. Rev. Lett. 113, 093603 (2014).

18. Hartschuh, A., Sánchez, E. J., Xie, X. S. \& Novotny, L. High-Resolution Near-Field Raman Microscopy of Single-Walled Carbon Nanotubes. Phys. Rev. Lett. 90, 095503 (2003).

19. Langer, J. et al. Present and Future of Surface-Enhanced Raman Scattering. ACS Nano 14, 28-117 (2020).

20. Schlücker, S. Surface-Enhanced Raman Spectroscopy: Concepts and Chemical Applications. Angew. Chem. Int. Ed. 53, 4756-4795 (2014).

21. Fleischmann, M., Hendra, P. J. \& McQuillan, A. J. Raman spectra of pyridine adsorbed at a silver electrode. Chem. Phys. Lett. 26, 163-166 (1974).

22. Jeanmaire, D. L. \& Van Duyne, R. P. Surface raman spectroelectrochemistry: Part I. Heterocyclic, aromatic, and aliphatic amines adsorbed on the anodized silver electrode. J. Electroanal. Chem. Interfacial Electrochem. 84, 1-20 (1977).

23. Kühler, P. et al. Plasmonic DNA-Origami Nanoantennas for Surface-Enhanced Raman Spectroscopy. Nano Lett. 14, 2914-2919 (2014).

24. Peyskens, F., Dhakal, A., Van Dorpe, P., Le Thomas, N. \& Baets, R. Surface Enhanced Raman Spectroscopy Using a Single Mode Nanophotonic-Plasmonic Platform. ACS Photonics 3, 102-108 (2016).

25. Chen, C. et al. Focusing Plasmons in Nanoslits for Surface-Enhanced Raman Scattering. Small 5, 2876-2882 (2009).

26. Lim, D.-K. et al. Highly uniform and reproducible surface-enhanced Raman scattering from DNAtailorable nanoparticles with 1-nm interior gap. Nat. Nanotechnol. 6, 452-460 (2011).

27. Peyskens, F., Wuytens, P., Raza, A., Dorpe, P. V. \& Baets, R. Waveguide excitation and collection of surface-enhanced Raman scattering from a single plasmonic antenna. Nanophotonics 7, 1299-1306 (2018).

28. Zhang, C. et al. Chemical mapping of a single molecule by plasmon-enhanced Raman scattering. Nature 498, 82 (2013). 
29. De Angelis, F. et al. Nanoscale chemical mapping using three-dimensional adiabatic compression of surface plasmon polaritons. Nat. Nanotechnol. 5, 67-72 (2010).

30. Kriesch, A. et al. Functional Plasmonic Nanocircuits with Low Insertion and Propagation Losses. Nano Lett. 13, 4539-4545 (2013).

31. Geisler, P. et al. Multimode Plasmon Excitation and In Situ Analysis in Top-Down Fabricated Nanocircuits. Phys. Rev. Lett. 111, 183901 (2013).

32. Huang, K. C. Y. et al. Electrically driven subwavelength optical nanocircuits. Nat. Photonics 8, 244249 (2014).

33. Lai, C.-H. et al. Near infrared surface-enhanced Raman scattering based on star-shaped gold/silver nanoparticles and hyperbolic metamaterial. Sci. Rep. 7, 5446 (2017).

34. Smith, R. G. Optical Power Handling Capacity of Low Loss Optical Fibers as Determined by Stimulated Raman and Brillouin Scattering. Appl. Opt. 11, 2489-2494 (1972).

35. Jun, Y. C., Kekatpure, R. D., White, J. S. \& Brongersma, M. L. Nonresonant enhancement of spontaneous emission in metal-dielectric-metal plasmon waveguide structures. Phys. Rev. B 78, 153111 (2008).

36. Genov, D. A., Oulton, R. F., Bartal, G. \& Zhang, X. Anomalous spectral scaling of light emission rates in low-dimensional metallic nanostructures. Phys. Rev. B 83, 245312 (2011).

37. Bozhevolnyi, S. I. \& Khurgin, J. B. Fundamental limitations in spontaneous emission rate of singlephoton sources. Optica 3, 1418-1421 (2016). 


\section{Supplementary Files}

This is a list of supplementary files associated with this preprint. Click to download.

- SupplementaryInformation.docx 